\title{
B physics beyond the Standard Model
}

\author{
A. Masiero and $\mathbf{O}$. Vives \\ SISSA/ISAS, Trieste, Italy, via Beirut 2-4, 34013 and INFN, Sezione di Trieste, Trieste, Italy \\ Email: 'masiero@sissa.it' and vives@sissa.it'
}

ABSTRACT: In this talk, we address the possibility of finding supersymmetry through indirect searches in the $B$ system. We prove that, in the absence of the Cabibbo-Kobayashi-Maskawa phase, a general Minimal Supersymmetric Standard Model with all possible phases in the soft-breaking terms, but no new flavor structure beyond the usual Yukawa matrices, can never give a sizeable contribution to $\varepsilon_{K}, \varepsilon^{\prime} / \varepsilon$ or hadronic $B^{0} \mathrm{CP}$ asymmetries. However, Minimal Supersymmetric models with additional flavor structures in the soft-supersymmetry breaking terms can produce large deviations from the Standard Model predictions. Hence, observation of supersymmetric contributions to CP asymmetries in B decays would be the first sign of the existence of new flavor structures in the soft-terms and would hint at a non-flavor blind mechanism of supersymmetry breaking.

\section{Introduction}

Beginning with its experimental discovery in $K-$ meson decays, about three decades ago, the origin of $\mathrm{CP}$ violation has been one of the most intriguing questions in particle phenomenology. Notably, the subsequent experiments in the search for electric dipole moments (EDM) of the neutron and electron have observed no sign of new $\mathrm{CP}$-violating effects despite their considerably high precision. Hence, the neutral $K$-system remains, so far, the only experimental information on the presence of $\mathrm{CP}$-violation in nature.

In the near future, this situation will change. Not only the new $B$ factories will start measuring $\mathrm{CP}$ violation effects in $B^{0} \mathrm{CP}$ asymmetries, but also the experimental sensitivity to the electric dipole moment of the neutron and the electron will be substantially improved. These new experiments will enlarge our knowledge of CP violation phenomena and, hopefully, will show the existence of new sources of CP violation from models beyond the Standard Model (SM).

The Standard Model of electroweak interactions is known to be able to accommodate the experimentally observed $\mathrm{CP}$-violation through a unique phase, $\delta_{C K M}$, in the Cabibbo-KobayashiMaskawa mixing matrix (CKM). However, most of the extensions of the SM include new observable phases that may significantly modify the pattern of CP violation. Supersymmetry is, with no doubt, one of the most popular extensions of the SM. Indeed, in the minimal supersymmetric extension of the SM (MSSM), there are additional phases which can cause deviations from the predictions of the SM. After all possible rephasings of the parameters and fields, there remain at least two new physical phases in the MSSM Lagrangian. These phases can be chosen to be the phases of the Higgsino Dirac mass parameter $\left(\varphi_{\mu}=\operatorname{Arg}[\mu]\right)$ and the trilinear sfermion coupling to the Higgs, $\left(\varphi_{A_{0}}=\operatorname{Arg}\left[A_{0}\right]\right)\left[\underline{1}\left[\begin{array}{l}1 \\ 1\end{array}\right.\right.$. In fact, in the so-called Constrained Minimal Supersymmetric Standard Model (CMSSM), with strict universality at the Grand Unification scale, these are the only new phases present.

It was soon realized, that for most of the MSSM parameter space, the experimental bounds on the electric dipole moments of the electron and neutron constrained $\varphi_{A_{0}, \mu}$ to be at most $\mathcal{O}\left(10^{-2}\right)$. Consequently these new supersymmetric phases have been taken to vanish exactly in most studies in the framework of the MSSM.

However, in the last few years, the possibility of having non-zero SUSY phases has again attracted a great deal of attention. Several new 
mechanisms have been proposed to suppress supersymmetric contributions to EDMs below the experimental bounds while allowing SUSY phases $\mathcal{O}(1)$. Methods of suppressing the EDMs consist of cancellation of various SUSY contributions

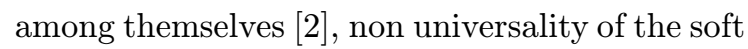
breaking parameters at the unification scale [30] and approximately degenerate heavy sfermions for the first two generations $[\overline{4}[\overline{4}]$. In the presence of one of these mechanisms, large supersymmetric phases are naturally expected and EDMs should be generally close to the experimental bounds.

In this work we will study the effects of these phases in CP violation observables as $\varepsilon_{K}, \varepsilon^{\prime} / \varepsilon$ and $B^{0} \mathrm{CP}$ asymmetries. We will show that the presence of large susy phases is not enough to produce sizeable supersymmetric contributions to these observables. In fact, in the absence of the CKM phase, a general MSSM with all possible phases in the soft-breaking terms, but no new flavor structure beyond the usual Yukawa matrices, can never give a sizeable contribution to $\varepsilon_{K}, \varepsilon^{\prime} / \varepsilon$ or hadronic $B^{0}$ CP asymmetries. However, as recently emphasized $[\overline{5}, \overline{3}, \overline{3} \mid$, as soon as one introduces some new flavor structure in the soft Susybreaking sector, even if the $\mathrm{CP}$ violating phases are flavor independent, it is indeed possible to get sizeable CP contribution for large Susy phases and $\delta_{C K M}=0$. Then, we can rephrase our sentence above in a different way: $A$ new result in hadronic $B^{0}$ CP asymmetries in the framework of supersymmetry would be a direct prove of the existence of a completely new flavor structure in the soft-breaking terms. This means that $B$-factories will probe the flavor structure of the supersymmetry soft-breaking terms even before the direct discovery of the supersymmetric partners [i's]

\section{Soft-breaking flavor structure}

As announced in the introduction, the presence of new flavor structure in the soft-breaking terms is necessary to obtain sizeable contributions to flavor-changing $\mathrm{CP}$ observables (i.e. $\varepsilon_{K}, \varepsilon^{\prime} / \varepsilon$ and hadronic $B^{0} \mathrm{CP}$ asymmetries). To prove this we will consider any MSSM, i.e. with the minimal supersymmetric particle content, with general complex soft-breaking terms, but with a flavor structure strictly given by the two familiar Yukawa matrices or any matrix strictly proportional to them. In these conditions, the most general structure of the soft-breaking terms at the large scale, that we call $M_{G U T}$, is,

$$
\begin{gathered}
\left(m_{Q}^{2}\right)_{i j}=m_{Q}^{2} \delta_{i j} \quad\left(m_{U}^{2}\right)_{i j}=m_{U}^{2} \delta_{i j} \\
\left(m_{D}^{2}\right)_{i j}=m_{D}^{2} \delta_{i j} \quad\left(m_{L}^{2}\right)_{i j}=m_{L}^{2} \delta_{i j} \\
\left(m_{E}^{2}\right)_{i j}=m_{E}^{2} \delta_{i j} \quad m_{H_{1}}^{2} \quad m_{H_{2}}^{2} \\
m_{\tilde{g}} e^{i \varphi_{3}} m_{\tilde{W}} e^{i \varphi_{2}} m_{\tilde{B}} e^{i \varphi_{1}} \\
\left(A_{U}\right)_{i j}=A_{U} e^{i \varphi_{A_{U}}}\left(Y_{U}\right)_{i j} \\
\left(A_{D}\right)_{i j}=A_{D} e^{i \varphi_{A_{D}}}\left(Y_{D}\right)_{i j} \\
\left(A_{E}\right)_{i j}=A_{E} e^{i \varphi_{A_{E}}}\left(Y_{E}\right)_{i j} .
\end{gathered}
$$

where all the allowed phases are explicitly written and one of them can be removed by an $\mathrm{R}-$ rotation. All other numbers or matrices in this equation are always real. Notice that this struc-

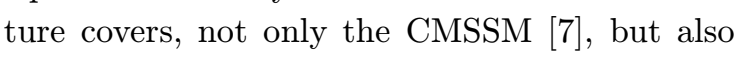
most of Type I string motivated models consid-

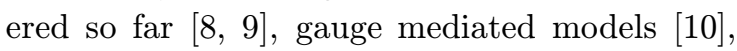
minimal effective supersymmetry models [11 $1,1,121$, etc.

Experiments of $\mathrm{CP}$ violation in the $K$ or $B$ systems only involve supersymmetric particles as virtual particles in the loops. This means that the phases in the soft-breaking terms can only appear in these experiments through the mass matrices of the susy particles. Then, the key point in our discussion will be the role played by the susy phases and the soft-breaking terms flavor structure in the low-energy sparticle mass matrices.

It is important to notice that, even in a model with flavor-universal soft-breaking terms at some high energy scale, as this is the case, some offdiagonality in the squark mass matrices appears at the electroweak scale. Working in the basis where the squarks are rotated parallel to the quarks, the so-called Super CKM basis (SCKM), the squark mass matrix is not flavor diagonal at $M_{W}$. This is due to the fact that at $M_{G U T}$ there are always two non-trivial flavor structures, namely the two Yukawa matrices for the up and down quarks, not simultaneously diagonalizable. This implies that through RGE evolution some flavor mixing leaks into the sfermion mass ma- 
trices. In a general Supersymmetric model, the presence of new flavor structures in the soft breaking terms would generate large flavor mixing in the sfermion mass matrices. However, in the CMSSM, the two Yukawa matrices are the only source of flavor change. Always in the SCKM basis, any off-diagonal entry in the sfermion mass matrices at $M_{W}$ will be necessarily proportional to a product of Yukawa couplings. Then, a typical estimate for the element $(i, j)$ in the $L-L$ down squark mass matrix at the electroweak scale would necessarily be (see [i], for details),

$$
\left(m_{L L}^{2(D)}\right)_{i j} \approx c m_{Q}^{2} Y_{i k}^{u} Y_{j k}^{u *},
$$

with $c$ a proportionality factor between 0.1 and 1 . This rough estimate provides the order of magnitude of the different entries in the sfermion mass matrices. It is important to notice that if the phases of these elements were $\mathcal{O}(1)$, due to some of the phases in equation $(\underline{\underline{2}} \cdot \overline{-}-\overline{1})$, we would be able to give sizeable contributions, or even saturate, the different CP observables [13in]. Then, it is clear that the relevant question for $\mathrm{CP}$ violation experiments is the presence of imaginary parts in these off-diagonal entries.

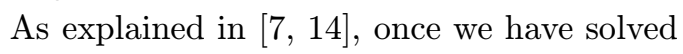
the Yukawa RGEs, the RGE equations of all softbreaking terms are a set of linear differential equations. Then, they can be solved as a linear function of the initial conditions,

$$
\begin{gathered}
m_{Q}^{2}\left(M_{W}\right)=\sum_{i} \eta_{Q}^{\left(\phi_{i}\right)} m_{\phi_{i}}^{2}+\sum_{i} \eta_{Q}^{\left(g_{i}\right)} m_{g_{i}}^{2} \\
+\sum_{i \neq j}\left(\eta_{Q}^{\left(g_{i j}\right)} e^{i \varphi_{i j}}+\eta_{Q}^{\left(g_{i j}\right) T} e^{-i \varphi_{i j}}\right) m_{g_{i}} m_{g_{j}} \\
+\sum_{i j}\left(\eta_{Q}^{\left(g A_{i j}\right)} e^{i \varphi_{i A_{j}}}+\eta_{Q}^{\left(g A_{i j}\right) T} e^{-i \varphi_{i A_{j}}}\right) m_{g_{i}} A_{j} \\
+\sum_{i \neq j}\left(\eta_{Q}^{\left(A_{i j}\right)} e^{i \varphi_{A_{i} A_{j}}}+\eta_{Q}^{\left(A_{i j}\right) T} e^{-i \varphi_{A_{i}} A_{j}}\right) A_{i} A_{j} \\
+\sum_{i} \eta_{Q}^{\left(A_{i}\right)} A_{i}^{2},
\end{gathered}
$$

where $\phi_{i}$ refers to any scalar, $g_{i}$ to the different gauginos, $A_{i}$ to any tri-linear coupling and the phases $\varphi_{a b}=\left(\varphi_{a}-\varphi_{b}\right)$. In this equation, the different $\eta$ matrices are $3 \times 3$ matrices, strictly real and all the allowed phases have been explicitly written. Regarding the imaginary parts, due to the hermiticity of the sfermion mass matrices, any imaginary part will always be associated to the non-symmetric part of the $\eta_{Q}^{\left(g_{i} g_{j}\right)}, \eta_{Q}^{\left(A_{i} A_{j}\right)}$ or $\eta_{Q}^{\left(g_{i} A_{j}\right)}$ matrices. To estimate the size of these anti-symmetric parts, we can go to the RGE equations for the scalar mass matrices, where we use the same conventions and notation as in [i], $\overline{1}_{1} \overline{1}_{4}$. Taking advantage of the linearity of these equations we can directly write the evolution of the anti-symmetric parts, $\hat{m}_{Q}^{2}=m_{Q}^{2}-\left(m_{Q}^{2}\right)^{T}$ as,

$$
\begin{aligned}
& \frac{d \hat{m}_{Q}^{2}}{d t}=-\left(\frac{1}{2}\left(\tilde{Y}_{U} \tilde{Y}_{U}^{\dagger}+\tilde{Y}_{D} \tilde{Y}_{D}^{\dagger}\right) \hat{m}_{Q}^{2}\right. \\
& \quad+\frac{1}{2} \hat{m}_{Q}^{2}\left(\tilde{Y}_{U} \tilde{Y}_{U}^{\dagger}+\tilde{Y}_{D} \tilde{Y}_{D}^{\dagger}\right) \\
& \quad+\tilde{Y}_{U} \hat{m}_{U}^{2} \tilde{Y}_{U}^{\dagger}+\tilde{Y}_{D} \hat{m}_{D}^{2} \tilde{Y}_{D}^{\dagger} \\
& \left.\quad+2 i \Im\left\{\tilde{A}_{U} \tilde{A}_{U}^{\dagger}+\tilde{A}_{D} \tilde{A}_{D}^{\dagger}\right\}\right),
\end{aligned}
$$

where, due to the reality of Yukawa matrices, we have used $Y^{T}=Y^{\dagger}$, and following [1]4 a tilde over the couplings $(\tilde{Y}, \tilde{A}, \ldots)$ denotes a rescaling by a factor $1 /(4 \pi)$. In the evolution of the $R-R$ squark mass matrices, $m_{U}^{2}$ and $m_{D}^{2}$, only one of the two Yukawa matrices, the one with equal isospin to the squarks, is directly involved. Then, it is easy to understand that these matrices are in a very good approximation diagonal in the SCKM basis once you start with the initial conditions given in equation (12.i). Hence, for the sake of clarity, we can safely neglect the last two terms in equation (2. and $\hat{m}_{D}^{2}$. However, if needed, we could always apply to estimate their anti-symmetric parts an analogous reasoning as the one we show below to $\hat{m}_{Q}^{2}$.

From equation (12.1), the initial conditions for $\hat{m}_{Q}^{2}$ at $M_{G U T}$ are identically zero. This means that the only source for $\hat{m}_{Q}^{2}$ in equation (2. necessarily $\Im\left\{A_{U} A_{U}^{\dagger}+A_{D} A_{D}^{\dagger}\right\}$.

The next step is then to analyze the RGE for the tri-linear couplings,

$$
\begin{gathered}
\frac{d \tilde{A}_{U}}{d t}=\frac{1}{2}\left(\frac{16}{3} \tilde{\alpha}_{3}+3 \tilde{\alpha}_{2}+\frac{1}{9} \tilde{\alpha}_{1}\right) \tilde{A}_{U} \\
-\left(\frac{16}{3} \tilde{\alpha}_{3} M_{3}+3 \tilde{\alpha}_{2} M_{2}+\frac{1}{9} \tilde{\alpha}_{1} M_{1}\right) \tilde{Y}_{U} \\
-\left(2 \tilde{A}_{U} \tilde{Y}_{U}^{\dagger} \tilde{Y}_{U}+3 \operatorname{Tr}\left(\tilde{A}_{U} \tilde{Y}_{U}^{\dagger}\right) \tilde{Y}_{U}\right. \\
+\frac{5}{2} \tilde{Y}_{U} \tilde{Y}_{U}^{\dagger} \tilde{A}_{U}+\frac{3}{2} \operatorname{Tr}\left(\tilde{Y}_{U} \tilde{Y}_{U}^{\dagger}\right) \tilde{A}_{U} \\
\left.\quad+\tilde{A}_{D} \tilde{Y}_{D}^{\dagger} \tilde{Y}_{U}+\frac{1}{2} \tilde{Y}_{D} \tilde{Y}_{D}^{\dagger} \tilde{A}_{U}\right)
\end{gathered}
$$

with an equivalent equation for $A_{D}$. With the general initial conditions in equation $(2.1), A_{U}$ is 
complex at any scale. However, we are interested in the imaginary parts of $A_{U} A_{U}^{\dagger}$. At $M_{G U T}$ this combination is exactly real, but, due to different renormalization of different elements of the matrix, this is not true any more at a different scale.

However, a careful analysis of equation (2. $(2,5)$ is enough to convince ourselves that these imaginary parts are extremely small. Let us, for a moment, neglect the terms involving $\tilde{A}_{D} \tilde{Y}_{D}^{\dagger}$ or $\tilde{Y}_{D} \tilde{Y}_{D}^{\dagger}$ from the above equation. Then, the only flavor structure appearing in equation (2. 2.5$)$ at $M_{G U T}$ is $Y_{U}$. We can always go to the basis where $Y_{U}$ is diagonal and then we will have $A_{U}$ exactly diagonal at any scale. In particular this means that $\Im\left\{A_{U} A_{U}^{\dagger}\right\}$ would always exactly vanish. A completely paralel reasoning can be applied to $A_{D}$ and $\Im\left\{A_{D} A_{D}^{\dagger}\right\}$. Hence, simply taking into account the flavor structure, our conclusion is that, necessarily, any non-vanishing element of $\Im\left[A_{U} A_{U}^{\dagger}+A_{D} A_{D}^{\dagger}\right]$ and hence of $\hat{m}_{Q}^{2}$ must be proportional to $\left(\tilde{Y}_{D} \tilde{Y}_{D}^{\dagger} \tilde{Y}_{U} \tilde{Y}_{U}^{\dagger}-H . C\right.$. $)$. So, we can expect them to be,

$$
\begin{aligned}
\left(\hat{m}_{Q}^{2}\right)_{i<j} & \approx K\left(Y_{D} Y_{D}^{\dagger} Y_{U} Y_{U}^{\dagger}-H . C .\right)_{i<j} \\
\left(\hat{m}_{Q}^{2}\right)_{12} & \approx K \cos ^{-2} \beta\left(h_{s} h_{t} \lambda^{5}\right) \\
\left(\hat{m}_{Q}^{2}\right)_{13} & \approx K \cos ^{-2} \beta\left(h_{b} h_{t} \lambda^{3}\right) \\
\left(\hat{m}_{Q}^{2}\right)_{23} & \approx K \cos ^{-2} \beta\left(h_{b} h_{t} \lambda^{2}\right),
\end{aligned}
$$

where $h_{i}=m_{i}^{2} / v^{2}$, with $v=\sqrt{v_{1}^{2}+v_{2}^{2}}$ the vacuum expectation value of the Higgs, $\lambda=\sin \theta_{c}$ and $K$ is a proportionality constant that includes the effects of the running from $M_{G U T}$ to $M_{W}$. To estimate this constant we have to keep in mind that the imaginary parts of $A_{U} A_{U}^{\dagger}$ are generated through the RGE running and then these imaginary parts generate $\hat{m}_{Q}^{2}$ as a second order effect. This means that roughly $K \simeq \mathcal{O}\left(10^{-2}\right)$ times a combination of initial conditions as in equation (2.3i). So, we estimate these matrix elements to be $\left(\cos ^{-2} \beta\left\{10^{-12}, 6 \times 10^{-8}, 3 \times 10^{-7}\right\}\right)$ times initial conditions. This was exactly the result we

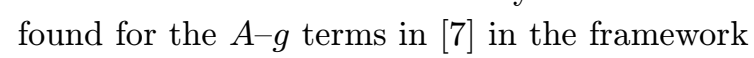
of the CMSSM. In fact, now it is clear that this is the same for all the terms in equation $(\overline{2} . \overline{3}), g_{i}$ $A_{j}, g_{i}-g_{j}$ and $A_{i}-A_{j}$, irrespectively of the presence of an arbitrary number of new phases.

As we have already said before, the situation in the $R-R$ matrices is still worse because the RGE of these matrices involves only the corresponding Yukawa matrix and hence, in the SCKM, they are always diagonal and real in extremely good approximation.

Hence, so far, we have shown that the $L-L$ or $R-R$ squark mass matrices are still essentially real. The only complex matrices, then, will still be the $L-R$ matrices that include, from the very beginning, the phases $\varphi_{A_{i}}$ and $\varphi_{\mu}$. Once more, the size of these entries is determined by the $\mathrm{Yu}$ kawa elements with these two phases providing the complex structure. However, this situation is not new for these more general MSSM models and it was already present even in the CMSSM. We can conclude, then, that the structure of the sfermion mass matrices at $M_{W}$ is not modified from the familiar structure already present in the CMSSM, irrespective of the presence of an arbitrary number of new susy phases.

In the next section we analyze the different indirect and direct $\mathrm{CP}$ violation observables in this general MSSM without new flavor structure.

\section{CP observables}

\subsection{Indirect CP violation}

In first place, we will consider indirect $\mathrm{CP}$ violation both in the $K$ and $B$ systems. In the SM neutral meson mixing arises at one loop through the well-known $W$-box. However, in the MSSM, there are new contributions to $\Delta F=2$ processes coming from boxes mediated by supersymmetric particles. These are: charged Higgs boxes $\left(H^{ \pm}\right)$, chargino boxes $\left(\chi^{ \pm}\right)$and gluino-neutralino boxes $\left(\tilde{g}, \chi^{0}\right) . \quad \mathcal{M}-\overline{\mathcal{M}}$ mixing is correctly described by the $\Delta F=2$ effective Hamiltonian, $\mathcal{H}_{\text {eff }}^{\Delta F=2}$, which can be decomposed as,

$$
\begin{gathered}
\mathcal{H}_{\text {eff }}^{\Delta F=2}=-\frac{G_{F}^{2} M_{W}^{2}}{(2 \pi)^{2}}\left(V_{t d}^{*} V_{t q}\right)^{2}\left(C_{1}(\mu) Q_{1}(\mu)\right. \\
\left.+C_{2}(\mu) Q_{2}(\mu)+C_{3}(\mu) Q_{3}(\mu)\right) .
\end{gathered}
$$

With the relevant four-fermion operators given by

$$
\begin{aligned}
Q_{1} & =\bar{d}_{L}^{\alpha} \gamma^{\mu} q_{L}^{\alpha} \cdot \bar{d}_{L}^{\beta} \gamma_{\mu} q_{L}^{\beta} \\
Q_{2} & =\bar{d}_{L}^{\alpha} q_{R}^{\alpha} \cdot \bar{d}_{L}^{\beta} q_{R}^{\beta} \\
Q_{3} & =\bar{d}_{L}^{\alpha} q_{R}^{\beta} \cdot \bar{d}_{L}^{\beta} q_{R}^{\alpha}
\end{aligned}
$$


where $q=s, b$ for the $K$ and $B$-systems respectively and $\alpha, \beta$ are color indices. In the CMSSM, these are the only three operators present in the limit of vanishing $m_{d}$. The Wilson coefficients, $C_{1}(\mu), C_{2}(\mu)$ and $C_{3}(\mu)$, receive contributions from the different supersymmetric boxes,

$$
\begin{aligned}
C_{1}\left(M_{W}\right) & =C_{1}^{W}\left(M_{W}\right)+C_{1}^{H}\left(M_{W}\right) \\
& +C_{1}^{\tilde{g}, \chi^{0}}\left(M_{W}\right)+C_{1}^{\chi}\left(M_{W}\right) \\
C_{2}\left(M_{W}\right) & =C_{2}^{H}\left(M_{W}\right)+C_{2}^{\tilde{g}}\left(M_{W}\right) \\
C_{3}\left(M_{W}\right) & =C_{3}^{\tilde{g}, \chi^{0}}\left(M_{W}\right)+C_{3}^{\chi}\left(M_{W}\right)
\end{aligned}
$$

Both, the usual SM $W$-box and the charged Higgs box contribute to these operators. However, with $\delta_{C K M}=0$, these contributions do not contain any complex phase and hence cannot generate an imaginary part for these Wilson coefficients.

Gluino and neutralino contributions are specifically supersymmetric. They involve the superpartners of quarks and gauge bosons. Here, the source of flavor mixing is not directly the usual CKM matrix. It is the presence of off-diagonal elements in the sfermion mass matrices, as discussed in section $\overline{2}$. From the point of view of CP violation, we will always need a complex Wilson coefficient. In the SCKM basis all gluino vertices are flavor diagonal and real. This means that in the mass insertion (MI) approximation, we need a complex MI in one of the sfermion lines. Only $L-L$ mass insertions enter at first order in the Wilson coefficient $C_{1}^{\tilde{g}, \chi^{0}}\left(M_{W}\right)$. From equation (2.6), the imaginary parts of these MI are at most $\mathcal{O}\left(10^{-6}\right)$ for the $b-s$ transitions and smaller otherwise [7, [in. Comparing these values with the phenomenological bounds required to saturate the measured values of these processes [1 13 in] we can easily see that in this model we are always several orders of magnitude below.

In the case of the Wilson coefficients $C_{2}{ }^{\tilde{g}}\left(M_{W}\right)$ and $C_{3}{ }^{\tilde{g}}\left(M_{W}\right)$, the MI are $L-R$. However these MI are always suppressed by light masses of right handed squark, or in the case of $b-s$ transitions directly constrained by the $b \rightarrow s \gamma$ decay. Hence, gluino boxes, in the absence of new flavor structures, can never give sizeable contributions to in-

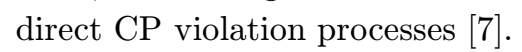

The chargino contributions to these Wilson coefficients were discussed in great detail in the

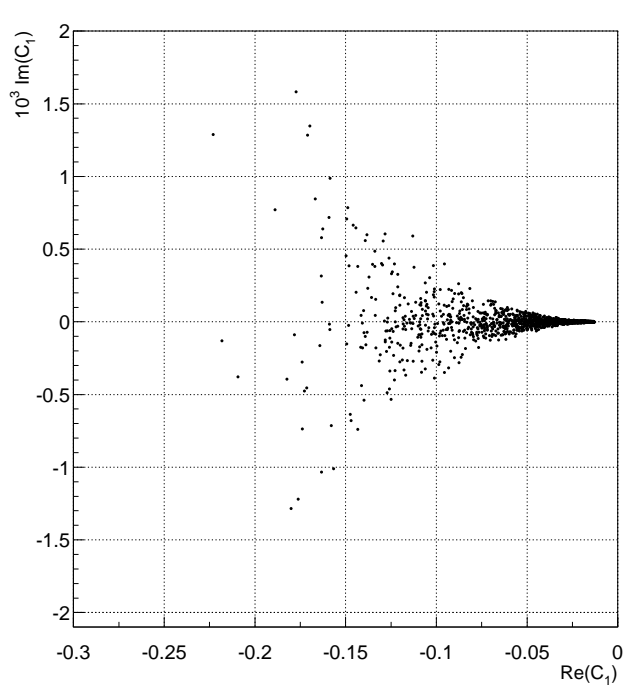

Figure 1: Imaginary and Real parts of the Wilson coefficient $C_{1}^{\chi}$ in B mixing.

CMSSM framework in reference [7і]. In this more general MSSM, as we have explained in section 2. we find very similar results due to the absence of new flavor structure.

Basically, in the chargino boxes, flavor mixing comes explicitly from the CKM mixing matrix, although off-diagonality in the sfermion mass matrix introduces a small additional source of flavor mixing.

$$
\begin{gathered}
C_{1}^{\chi}\left(M_{W}\right)=\sum_{i, j=1}^{2} \sum_{k, l=1}^{6} \sum_{\alpha \gamma \alpha^{\prime} \gamma^{\prime}} \\
\frac{V_{\alpha^{\prime} d}^{*} V_{\alpha q} V_{\gamma^{\prime} d}^{*} V_{\gamma q}}{\left(V_{t d}^{*} V_{t q}\right)^{2}} G^{(\alpha, k) i} G^{\left(\alpha^{\prime}, k\right) j *} \\
G^{\left(\gamma^{\prime}, l\right) i *} G^{(\gamma, l) j} \quad Y_{1}\left(z_{k}, z_{l}, s_{i}, s_{j}\right)
\end{gathered}
$$

where $V_{\alpha q} G^{(\alpha, k) i}$ represent the coupling of chargino and squark $k$ to left-handed down quark $q$, $z_{k}=M_{\tilde{u}_{k}}^{2} / M_{W}^{2}$ and $s_{i}=M_{\tilde{\chi}_{i}}^{2} / M_{W}^{2}$. The explicit expressions for these couplings and loop functions can be found in reference $\left[\overline{\bar{T}_{1}}\right] . G^{(\alpha, k) i}$ are in general complex, as both $\varphi_{\mu}$ and $\varphi_{A_{i}}$ are present in the different mixing matrices.

The main part of $C_{1}^{\chi}$ in equation (3.4) will be given by pure CKM flavor mixing, neglecting the additional flavor mixing in the squark mass matrix $\left[1 \overline{5}_{1}^{1}, 1{ }_{1}\right]$. This means, $\alpha=\alpha^{\prime}$ and $\gamma=\gamma^{\prime}$. In these conditions, using the symmetry of loop function $Y_{1}(a, b, c, d)$ under the exchange of any two indices it is easy to prove that $C_{1}^{\chi}$ would be exactly real [1] $1_{1}$. This is not exactly true either in 


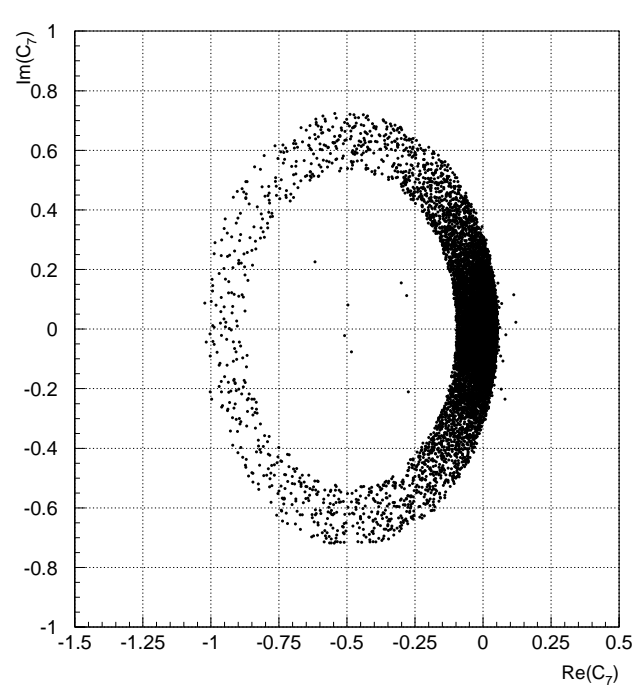

Figure 2: Experimental constraints on the Wilson coefficient $\mathcal{C}_{7}$.

the CMSSM or in our more general MSSM, where there is additional flavor change in the sfermion mass matrices. Here, some imaginary parts appear in the $C_{1}^{\chi}$ in equation $(3.4)$. In figure show in a scatter plot the size of imaginary and real parts of $C_{1}^{\chi}$ in the B system for a fixed value of $\tan \beta=40$. We see that this Wilson coefficient is always real up to a part in $10^{3}$. In any case, this is out of reach for the foreseen $\mathrm{B}$-factories.

Finally, chargino boxes contribute also to the quirality changing Wilson coefficient $C_{3}^{\chi}\left(M_{W}\right)$,

$$
\begin{gathered}
C_{3}^{\chi}\left(M_{W}\right)=\sum_{i, j=1}^{2} \sum_{k, l=1}^{6} \sum_{\alpha \gamma \alpha^{\prime} \gamma^{\prime}} \\
\frac{V_{\alpha^{\prime} d}^{*} V_{\alpha q} V_{\gamma^{\prime} d}^{*} V_{\gamma q}}{\left(V_{t d}^{*} V_{t q}\right)^{2}} \frac{m_{q}^{2}}{2 M_{W}^{2} \cos ^{2} \beta} \\
H^{(\alpha, k) i} G^{\left(\alpha^{\prime}, k\right) j *} G^{\left(\gamma^{\prime}, l\right) i *} H^{(\gamma, l) j} \\
Y_{2}\left(z_{k}, z_{l}, s_{i}, s_{j}\right)
\end{gathered}
$$

where $m_{q} /\left(\sqrt{2} M_{W} \cos \beta\right) \cdot V_{\alpha q} \cdot H^{(\alpha, k) i}$ is the coupling of chargino and squark to the right-handed down quark $q$ i 7 . Unlike the $C_{1}^{\chi}$ Wilson coefficient, due to the differences between $H$ and $G$ couplings, $C_{3}^{\chi}$ is complex even in the absence of intergenerational mixing in the sfermion mass matrices [1 $\left.1 \overline{1}_{1}^{1}\right]$. Then, the presence of flavor violating entries in the up-squark mass matrix hardly modifies the results obtained in their absence $\left[1 \overline{F_{1}}\right.$, $\overline{1} \overline{1}, \overline{1}, \overline{7}]$. In fact, in spite the presence of the Yukawa coupling squared, $m_{q}^{2} /\left(2 M_{W}^{2} \cos ^{2} \beta\right)$, this contribution could be relevant in the large $\tan \beta$ regime. For instance, in $B^{0}-\bar{B}^{0}$ mixing we have $m_{b}^{2} /\left(2 M_{W}^{2} \cos ^{2} \beta\right)$ that for $\tan \beta \gtrsim 25$ is larger than 1 and so, it is not suppressed at all when compared with the $C_{1}^{\chi}$ Wilson Coefficient. This means that this contribution can be very important in the large $\tan \beta$ regime [1] $\left.\overline{1}_{1}^{\prime}\right]$ and could have observable effects in $\mathrm{CP}$ violation experiments in the new B-factories. However, we will show next, that when we include the constraints coming from $b \rightarrow s \gamma$ these chargino contributions are also reduced to an unobservable level.

The chargino contributes to the $b \rightarrow s \gamma$ decay through the Wilson coefficients $\mathcal{C}_{7}$ and $\mathcal{C}_{8}$, corresponding to the photon and gluon dipole penguins respectively $\left[\overline{1} \overline{4}_{v}^{1}, \overline{1} \overline{7}_{1}, \overline{7}_{1}\right]$. In the large $\tan \beta$ regime, we can approximate these Wilson coefficients as [īi],

$$
\begin{aligned}
& \mathcal{C}_{7}^{\chi^{ \pm}}\left(M_{W}\right)=\sum_{k=1}^{6} \sum_{i=1}^{2} \sum_{\alpha, \beta=u, c, t} \frac{V_{\alpha b} V_{\beta s}^{*}}{V_{t b} V_{t s}^{*}} \\
& \frac{m_{b}}{\sqrt{2} M_{W} \cos \beta} H^{(\alpha, k) i} G^{*(\beta, k) i} \frac{M_{\chi^{i}}}{m_{b}} F_{R}^{7}\left(z_{k}, s_{i}\right) \\
& \mathcal{C}_{8}^{\chi^{ \pm}}\left(M_{W}\right)=\sum_{k=1}^{6} \sum_{i=1}^{2} \sum_{\alpha, \beta=u, c, t} \frac{V_{\alpha b} V_{\beta s}^{*}}{V_{t b} V_{t s}^{*}} \\
& \frac{m_{b}}{\sqrt{2} M_{W} \cos \beta} H^{(\alpha, k) i} G^{*(\beta, k) i} \frac{M_{\chi^{i}}}{m_{b}} F_{R}^{8}\left(z_{k}, s_{i}\right)
\end{aligned}
$$

Now, if we compare the chargino contributions to these Wilson coefficients and to the coefficient $C_{3}$, equations $(\overline{3} . \overline{5})$ and $(\overline{3} . \overline{6})$, we can see that they are deeply related. In fact, in the approximation where the two different loop functions involved are of the same order, we have,

$$
C_{3}\left(M_{W}\right) \approx\left(\mathcal{C}_{7}\left(M_{W}\right)\right)^{2} \frac{m_{q}^{2}}{M_{W}^{2}}
$$

In figure $\overline{2}$, we show a scatter plot of the allowed values of $\operatorname{Re}\left(\mathcal{C}_{7}\right)$ versus $\operatorname{Im}\left(\mathcal{C}_{7}\right)$ in the

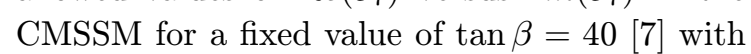
the constraints from the decay $B \rightarrow X_{s} \gamma$ taken from the reference [1] ${ }^{1}$. Notice that a relatively large value of $\tan \beta$, say $\tan \beta \gtrsim 10$, is needed to compensate the $W$ and charged Higgs contributions and cover the whole allowed area with positive and negative values. However, the shape of the plot is clearly independent of $\tan \beta$, only the number of allowed points and its location in the allowed area depend on the value con- 


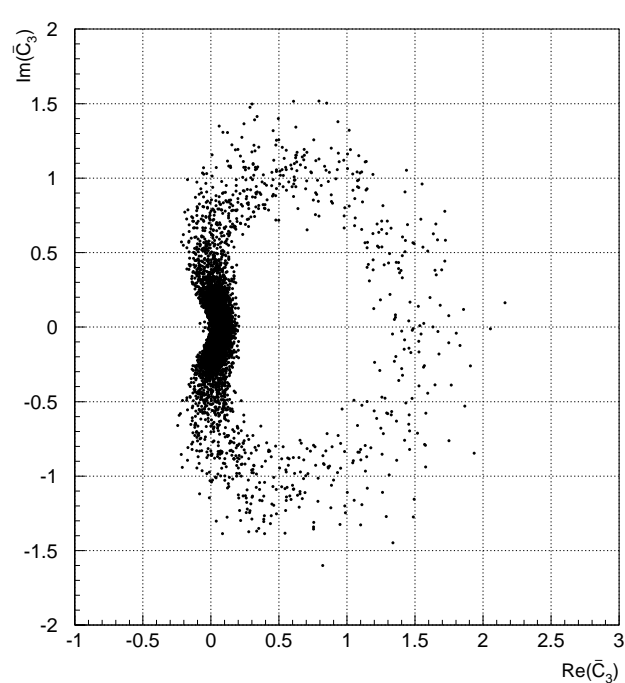

Figure 3: Allowed values for the re-scaled WC $\bar{C}_{3}$.

sidered. Then, figure $\overline{3}-1$ shows the allowed values for a re-scaled Wilson coefficient $\bar{C}_{3}\left(M_{W}\right)=$ $M_{W}^{2} / m_{q}^{2} C_{3}\left(M_{W}\right)$ corresponding to the same allowed points of the susy parameter space in figure [1. As we anticipated previously, the allowed values for $\bar{C}_{3}$ are close to the square of the values of $\mathcal{C}_{7}$ in figure $\overline{2}_{1}^{1}$ slightly scaled by different values of the loop functions.

We can immediately translate this result to a constraint on the size of the chargino contributions to $\varepsilon_{\mathcal{M}}$.

$$
\begin{gathered}
\varepsilon_{\mathcal{M}}=\frac{G_{F}^{2} M_{W}^{2}}{4 \pi^{2} \sqrt{2} \Delta M_{\mathcal{M}}} \frac{\left(V_{t d} V_{t q}\right)^{2}}{24} \\
F_{\mathcal{M}}^{2} M_{\mathcal{M}} \frac{M_{\mathcal{M}}^{2}}{m_{q}^{2}(\mu)+m_{d}^{2}(\mu)} \\
\eta_{3}(\mu) B_{3}(\mu) \operatorname{Im}\left[C_{3}\right]
\end{gathered}
$$

In this expression $M_{\mathcal{M}}, \Delta M_{\mathcal{M}}$ and $F_{\mathcal{M}}$ denote the mass, mass difference and decay constant of the neutral meson $\mathcal{M}^{0}$. The coefficient $\eta_{3}(\mu)=$ 2.93 [119"] includes the RGE effects from $M_{W}$ to the meson mass scale, $\mu$, and $B_{3}(\mu)$ is the $\mathrm{B}_{-}^{-}$ parameter associated with the matrix element of the $Q_{3}$ operator [1 $\left.\overline{1}^{9}\right]$.

For the $K$ system, using the experimentally measured value of $\Delta M_{K}$ we obtain,

$$
\begin{aligned}
\varepsilon_{K}^{\chi} & =1.7 \times 10^{-2} \frac{m_{s}^{2}}{M_{W}^{2}} \operatorname{Im}\left[\bar{C}_{3}\right] \\
& \approx 0.4 \times 10^{-7} \operatorname{Im}\left[\bar{C}_{3}\right]
\end{aligned}
$$

Given the allowed values of $\bar{C}_{3}$ in figure $\overline{3}_{1}$, this means that in the MSSM, even with large susy phases, chargino cannot produce a sizeable contribution to $\varepsilon_{K}$.

The case of $B^{0}-\bar{B}^{0}$ mixing has a particular interest due to the arrival of new data from the B-factories. In fact, in the large $\tan \beta$ regime chargino contributions to indirect $\mathrm{CP}$ violation can be very important. However, for any value of $\tan \beta$, we must satisfy the bounds from the $b \rightarrow$ $s \gamma$ decay. Then, if we apply these constraints to the $B^{0}-\bar{B}^{0}$ mixing,

$$
\begin{gathered}
\varepsilon_{B}^{\chi}=0.17 \frac{m_{b}^{2}}{M_{W}^{2}} \operatorname{Im}\left[\bar{C}_{3}\right] \\
\approx 0.5 \times 10^{-3} \operatorname{Im}\left[\bar{C}_{3}\right]
\end{gathered}
$$

where once again, with the allowed values of figure $\overline{3}$ we get a very small contribution to $\mathrm{CP}$ violation in the mixing. We must take into account that the mixing-induced $\mathrm{CP}$ phase, $\theta_{M}$, measurable in $B^{0} \mathrm{CP}$ asymmetries, is related to $\varepsilon_{B}$ by $\theta_{M}=\arcsin \left\{2 \sqrt{2} \cdot \varepsilon_{B}\right\}$. The expected sensitivities on the $\mathrm{CP}$ phases at the $\mathrm{B}$ factories are around \pm 0.1 radians, so this supersymmetric chargino contribution will be absolutely out of reach.

\subsection{Direct CP violation}

To complete our analysis, we consider now direct $\mathrm{CP}$ violation. In this case, the different decay processes are described by a $\Delta F=1$ effective Hamiltonian. A complete operator basis for these transitions in a general MSSM involves 14 different operators [1에. The main difference with the case of indirect $\mathrm{CP}$ violation is that these operators receive contributions both from box and penguin diagrams. Nevertheless the discussion of the presence of imaginary is completely analogous to the case of indirect $\mathrm{CP}$ violation.

Once more, in the gluino case, $L-L$ transitions are real to a very good approximation, and several orders of magnitude below the phenomenological bounds [13 $L-R$ transitions are suppressed by quark masses or $b \rightarrow s \gamma$ decay. This is always true for the squark mass matrices obtained in section $\overline{2_{0}^{\prime}}$ and valid both for boxes and penguins.

Finally we are left with chargino contributions. The analysis of chargino boxes is exactly 
the same as in the previous section. In fact, even the Wilson coefficients are identical once we factor out the CKM elements. Then, for the penguins, $L-L$ transitions are exactly real if we neglect inter-generational mixing in the squark mass matrices. Taking into account this small mixing we find, for the very same reasons as in the indirect $\mathrm{CP}$ violation case, that imaginary parts are far too small. The relation of the $b \rightarrow s \gamma$ decay with the $L-R$ chargino penguins is even more transparent than before.

However, there is still one possibility to observe the effects of the new supersymmetric phases in the absence of new flavor structure. We have seen that the reason for the smallness of the contributions of chargino $L-R$ transitions is the experimental bound from the $B \rightarrow X_{s} \gamma$ branching ratio. This bound makes the chirality changing transitions, although complex, too small to compete with $L-L$ transitions. Hence, in these conditions, just the processes where only chirality changing operators contribute (EDMs or $b \rightarrow$ $s \gamma$ ), or observables where chirality flip operators are relevant $\left(b \rightarrow s l^{+} l^{-}\right)$can show the effects of

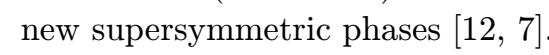

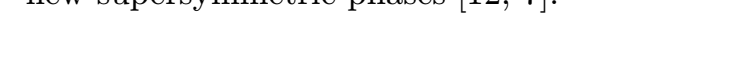

;.- - Conchusions

To_conclude _we_would like _to summmarizize_the posibilities of finding_supersymmetric_contribution - in-the-different CP. violation experiments.- - - - - - - - Irt the-presence of large supersymmetric pha , Ses, the_EDMM '- must be_very _close_to_the_experimental_bound - and possibly reachable-for the new generation-of

- experinents.- Hewever, as we-have shewn in this- work, the presence- of-these phases is not-enough-

- to generate a-sizeabte- contribution ${ }^{-10}{ }^{-} \varepsilon_{\bar{K}} ; \xi^{-} / \varepsilon$ - - r- $B^{0}$ - CP- asymmetries. - -n -this flaver- €hang- ing-EP-observables, the-presenee of a completely- - : -new- flavor structure in the soft breaking terms is

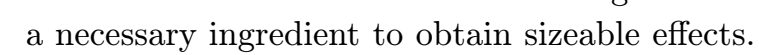

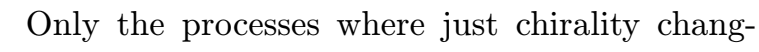
ing operators contribute as $b \rightarrow s \gamma$, or processes where these chirality flip operators are relevant, can show the effects of the new supersymmetric phases.

Nevertheless, in the presence of new flavor structure in the soft Susy-breaking sector it is indeed possible to get sizeable $\mathrm{CP}$ contribution with large Susy phases, even with $\delta_{C K M}=0$ [inj]. Then, a new result in hadronic $B^{0} \mathrm{CP}$ asymmetries in the framework of supersymmetry would be a direct prove of the existence of a completely new flavor structure in the soft-breaking terms. And so, $B$-factories will probe the flavor structure of the supersymmetry soft-breaking terms even before the direct discovery.

\section{Acknowledgments}

We thank D.A. Demir as the co-author of several works in which this talk is based and S. Bertolini, T. Kobayashi and S. Khalil for enlightening discussions. The work of A.M. was partially supported by the European TMR Project "Beyond the Standard Model" contract N. ERBFMRX CT96 0090; O.V. acknowledges financial support from a Marie Curie EC grant (TMR-ERBFMBI CT98 3087).

\section{References}

[1] M. Dugan, B. Grinstein and L.J. Hall, 'Nucl.' Phys. B 255 (1985) 413; S. Dimopoulos and S. Thomas, Nucl. Phys. B $465(1996) 23$, inep-ph/9510220

[2] T. Ibrahim and P. Nath, 'Phys. Rev. D -58' (1998) 111301, hep-ph/9807501;

M. Brhlik G.J. Good and G.L Kane Phys Rev. D_59 (1999) 115004 hep-ph/9810457!

[3] S.A. Abel and J.M. Frere, 'Phys. Rev. D 55' (1997) 1623; hep-ph/9608251!

S. Khalil, T. Kobayashi and A. Masiero, iPhys.' S Rev. D 60 (1999) 075003, hep-ph/9903544

S. Khalil and T. Kobayashi, Phys. Lett. B $\mathbf{4 6 0}$ (1999) 341, hep-ph/9906374

[4] S. Dimopoulos and G.F. Giudice, 'Phys. Lett.' В 357 (1995) 573, hep-ph/9507282; A. Cohen, D.B. Kaplan and A.E. Nelson, IPhys.' Lett. B $388(1996) 599$, hep-ph/9607394; A. Pomarol and D. Tommasini, $\mathbf{N}$ ucl. Phys. B' $466(1996)$ 3isep-ph/9507462i

[5] M. Brhlik, L. Everett, G.L. Kane, S.F. King and O. Lebedev, Virginia Pol. Inst. Report no. VPI/IPPAP/99/08, Sept. 1999, hep-ph/9909480'; A. Masiero and H. Murayama, 'Phys. Rev. Lett.' 83 (1999) 907, ihep-ph/9903363, 
R. Barbieri, R. Contino and A. Strumia, Pisa - - - - R. Barbieri, R. Contino and A. Strumia, Pisa ihep-ph/9908255,

[6] D.A. Demir, A. Masiero and O. Vives, SISSA report n. SISSA/134/99/EP hep-ph/9911337!

[7] D.A. Demir, A. Masiero and O. Vives, SISSA report n. SISSA/107/99/EP, accepted for publication in Phys. Rev. D, hep-ph/9909325i

[8] L.E. Ibanez, C. Munoz and S. Rigolin, 'Nucl.'.' ' - - - Phys. B 553 (1999) 43, hep-ph/9812397!

[9] M. Brhlik, L. Everett, G.L. Kane and J. Lykken, 'Phys. Rev. Lett. 83 (1999) 2124', hep-ph/9905215

M. Brhlik, L. Everett, G.L. Kane and J. Lykken, Fermilab preprint no. FERMILABPUB-99-230-T, August 1999, hep-ph/9908326, T. Ibrahim and P. Nath, Santa Barbara U. preprint no. NSF-ITP-99-129, October 1999, hep-ph/9910553'

[10] G.F. Giudice and R. Rattazzi, submitted to Phys. Rep., hep-ph/9801271', and references thererein.

[11] D.A. Demir, A. Masiero and O. Vives, iPhys.' '- Rev. Lett. 82 (1999) 2447, 'hep-ph/9812337!

[12] S. Baek and P. Ko, 'Phys. Rev. Lett. 83 (1999), ;

S. Baek and P. Ko, Korea Inst. Sci Report No. KAIST-TH-99-1, 1999, hep-ph/9904283.

[13] F. Gabbiani, E. Gabrielli, A. Masiero and L. Silvestrini, 'Nucl. Phys. B 477_(1996)_321', hep-ph/9604387'

[14] S. Bertolini, F. Borzumati, A. Masiero and G. Ridolfi, 'Nucl. Phys. B $\mathbf{3 5 3}$ (1991) 591!

[15] P. Cho, M. Misiak and D. Wyler, 'Phys. Rev.' ' - _ _ D 54 (1996) 3329' inep-ph/9601360!

[16] G.C. Branco, G.C. Cho, Y. Kizukuri and N. Oshimo, 'Nucl. Phys. B $\mathbf{4} 4 \mathbf{9}(1995)$ 483;

G.C. Branco, G.C. Cho, Y. Kizukuri and N. Oshimo, Phys. Lett. B $\mathbf{3 3 7}(1994) 316$ hep-ph/9408229!

[17] F.M. Borzumati, 'Z. Physik C 63 (1994) 291', hep-ph/9310212, S. Bertolini and F. Vissani, ZZ. Physik C $\mathbf{6 7}$ :- - $(1995)-513$, hep-ph/9403397;

T. Goto, Y.Y. Keum, T. Nihei, Y. Okada and Y. Shimizu, iPhys. Lett. B 460 (1999) 333', hep-ph/9812369!

[18] A.L. Kagan and M. Neubert, Eur. Phys. J. C7 (1999) 5 , hep-ph/9805303, 